\title{
Measurement Method of Lesion Area Using Adaptive Seeded Region Growing
}

\author{
Feng Guo \\ Department of Computer Science \\ Wenzheng College of Soochow University \\ Suzhou 215006, China \\ greedysky@163.com
}

\author{
Jian Wu, Yanqin Zhu \\ School of Computer Science and Technology \\ Soochow University \\ Suzhou 215006, China
}

\begin{abstract}
In order to measure the lesion area of the medicine images, this study proposes a method of the lesion area measurement which is based on the adaptive seeded region growing algorithm. The method manages optimization treatment of the threshold on the common seeded region growing, so as to avoid the phenomena of the insufficient segmentation or the excessive segmentation of the regional growing as far as possible, and enhance the accuracy of the regional segmentation. On that basis, the analytic method will be used to measure the area of the lesions, which has a good performance in the measurement of irregular region. Therefore, the simulation in this study reveals that it can locate the lesions region rapidly and correctly, and calculate the area of the lesion accurately.
\end{abstract}

Keywords-medical image;adaptive seeded region growing; seeded growing; irregular area; calculation of the area (key words)

\section{INTRODUCTION}

In modern medicine, it is doctors' regular work to measure and calculate the lesions area. Doctors have been using the traditional method to measure and identify the regional boundary of the heart and thoracic by using pens, rulers and films. After that they figure the approximate value by hand or with the help of calculator, and then to make the diagnosis. However, this traditional method is time-consuming and it often distains the film, and sometimes it is inaccurate. Fortunately, with the development of computer image processing technology, people find a new means for the accurate calculation of lesions area of medical images [1-3]. What's more, this new method improves the work efficiency and quality of the image diagnosis.

In this study, we will introduce the measurement of the lesions area based on the adaptive seeded region growing algorithm [4]. By using this, the things doctors need to do is click the lesions region they want to measure, and the algorithm can find the regional boundary automatically. Besides, some modification has been made on optimization treatment which can reduce the probability of insufficient segmentation and excessive segmentation, and is better than general region growing method. What's more, it improves the accuracy of lesions region and can measure and calculate the lesions area rapidly and accurately. The biggest advantage of this method is that it greatly improves doctor's work efficiency and avoids contamination of the films at the same time.

\section{THE PRINCIPLE OF REGIONAL GROWING}

In this study, we take the method of seeded region growing (SRG). Region growing segmentation is a kind of ancient segmentation method in image segmentation, and it was raised by Adams and Bischof [5]. The basic idea is that putting the pixels together to constitute a region, all pixels in region has the similar quality and characteristics. Begin with original region (center on selected seeds' pixel value), and expand region gradually by merging the neighboring pixel with similar quality and characteristics or other constituted regions to the current region. This progress continues until no pixel satisfying the condition is available.

There is an example about eight-connected [6-7] areas region growing method. In Fig .1(a), which pixel value's initialization gray scale value is 7 is regarded as the seed. From seed to the area of each pixel value, and take the absolute value from the difference between the seed pixel value and neighboring pixel value. When the absolute value is less than a certain threshold $\mathrm{T}$, this neighboring pixel will grow into a new seed and extend to every pixel around. Fig .1(b) shows the result of region growing when T's value is 3 . As can be seen from that we can see the circumambient pixels are all included in the growing region when their gray scale values are 6,5and 4. Fig .1(c) demonstrates the result of region growing when T's value is 4. Similarly, Fig .1(d) is the result when T's value is 5, and Fig .1(e) represents the result when T's value is 6. By that analogy, Fig .1(f) is the result of region growing when T's value is 7 . From all of these, we can see that whole matrix is divided into a region completely

$$
\left|\begin{array}{lllll}
0 & 1 & 5 & 6 & 2 \\
2 & 5 & 4 & 2 & 3 \\
4 & 3 & 7 & 0 & 2 \\
6 & 5 & 3 & 2 & 1 \\
6 & 0 & 1 & 4 & 4
\end{array}\right|\left|\begin{array}{lllll}
0 & 1 & 7 & 7 & 2 \\
2 & 7 & 7 & 2 & 3 \\
7 & 3 & 7 & 0 & 2 \\
7 & 7 & 3 & 2 & 1 \\
7 & 0 & 1 & 4 & 4
\end{array}\right|
$$

(b) $\mathrm{T}=3$ 


$\left|\begin{array}{lllll}0 & 1 & 7 & 7 & 2 \\ 2 & 7 & 7 & 2 & 7 \\ 7 & 7 & 7 & 0 & 2 \\ 7 & 7 & 7 & 2 & 1 \\ 7 & 0 & 1 & 7 & 7\end{array}\right|\left|\begin{array}{lllll}0 & 1 & 7 & 7 & 7 \\ 7 & 7 & 7 & 7 & 7 \\ 7 & 7 & 7 & 0 & 7 \\ 7 & 7 & 7 & 7 & 1 \\ 7 & 0 & 1 & 7 & 7\end{array}\right|$

(c) $\mathrm{T}=4$

$\left|\begin{array}{lllll}0 & 7 & 7 & 7 & 7 \\ 7 & 7 & 7 & 7 & 7 \\ 7 & 7 & 7 & 0 & 7 \\ 7 & 7 & 7 & 7 & 7 \\ 7 & 0 & 7 & 7 & 7\end{array}\right|\left|\begin{array}{lllll}7 & 7 & 7 & 7 & 7 \\ 7 & 7 & 7 & 7 & 7 \\ 7 & 7 & 7 & 7 & 7 \\ 7 & 7 & 7 & 7 & 7 \\ 7 & 7 & 7 & 7 & 7\end{array}\right|$

(e) $\mathrm{T}=6$

(f) $\mathrm{T}=7$

Figure 1. the example of eight-connected region growing

So it is very important to the selection of threshold T, In practical use, we need to deal with three questions of seeded region growing:

1) Select one of the start-point in the image as the seed.

2) Extend from the neighboring eight directions of the seed. When the absolute value of gray scale value difference between neighboring pixel and current seed is less than a certain threshold $\mathrm{T}$, then put this neighboring pixel to the current region.

3) When none of pixel which satisfies the condition is available, stop growing.

Region growing is one of serial region segmentation in image region segmentation. Its advantage is that the basic idea is relatively simple. Usually, it can segment the connected domain which shares the same quality and characteristics, and can provide enough boundary information and segmentation result. Region growing is able to satisfy various requirements at image segmentation, such as, it can not only aim at the special region to segment but also segment complex images like color images. Keys of region growing are the selection of appropriate seed and rule of growth. Rule of growth is made according to different principles, and different principles grow different regions. There are three common growing principles. For instance, one of these principles is based on region gray difference, one of these principles is based on statistical characteristics of gray distribution, and the other one is based on region shape [7-9]. In this study, we accept the first principle.

\section{THE MEASUREMENT OF LESION REGION}

\section{A. Segmenting regions}

Through the human-computer interaction, we can get a seed pixel when users click a point on the image. And then the pixel seed will grow from eight neighboring directions when it has the growing condition that it shares the connectivity and similarity with the seed point pixel. If the neighboring pixel meets the conditions, it will take the place of the original seed and become the new seed. The new seed will keep growing until it no long satisfies the growing condition. The steps are summarized as follows.

1) Get a seed pixel $P$ when the user clicks a certain point on the image, and the seed pixel first will be pushed onto the stack. Regard this point's gray scale value as $\mathrm{G}$, set the threshold as $\mathrm{T}$, and put this pixel point as the growing condition.

2) When the stack is not empty, pop the pixel element on the top of stack, and mark it as a special value in order to reduce cycled times.

3) Put pixel $P$ as the center, and consider the neighboring pixel $\mathrm{P}^{\prime}$. If $\mathrm{P}$ ' satisfies rule of growth (when absolute value of the difference between seed pixel value $\mathrm{P}$ and neighboring pixel value $\mathrm{P}$ ' is less than the threshold $\mathrm{T}$ ), merge $\mathrm{P}$ and $\mathrm{P}^{\text {' into }}$ new region, and push the new pixel $\mathrm{P}^{\prime}$ onto the stack at the same time.

4) Repeat step 2, until the stack is empty (there is no new seed goes in to stack). At this time, region stops growing.

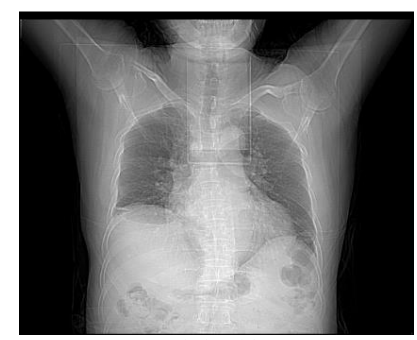

(a) Original image

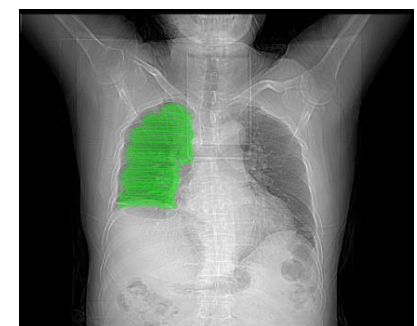

(b)Region growing segmentation image, when the threshold $\mathrm{T}$ is 50

Figure 2. the result of region growing segmentation

Fig .2(a) is the original medicine image before segmentation. Fig .2(b) is the image after region growing segmentation. According to the image segmentation result, we find that the algorithm has a great effect on medical image segmentation. But it has the problem of the insufficient segmentation which does not reach a satisfactory segmentation results. The key problem is related to the threshold $\mathrm{T}$ that we have selected, the current rule of growth is completely dependent on the selected threshold T. Therefore, we need to process optimization treatment of the current threshold on seeded region growing, so as to reduce the dependence on threshold T.

\section{B. Pretreating threshold}

As a result of the common seed region growing has limitations in image segmentation. Therefore, we need to deal with the original one. Taking into account limitations 
of the original seed region growing is mainly due to the threshold, so here we are mainly carries on processing to threshold. Up to now, there are a lot of methods to deal with it. Threshold segmentation is divided into the global threshold method and the local threshold segmentation method [10].

The local threshold segmentation method is that putting the original image into many smaller parts, and selecting the corresponding threshold value for each child images. Local threshold segmentation method is often used with gray - difference histogram and differential histogram method. The global threshold segmentation method uses a fixed threshold segmentation image across the entire image. According to the different threshold selection, the Global threshold segmentation can be divided into maximum between-cluster variance method, iterative threshold selection method and modal method.

The pretreatment process is to find out the optimal threshold in the medical image, the most used method is maximum between-cluster variance (OSTU) [11-12]. Its basic idea is to use a threshold to divide the whole data into two classes, if the variance between the two classes is the maximum, then this threshold is the optimal threshold.

Assume that the size of divided image is M x N. Set the foreground and background segmentation threshold as $\mathrm{T}$, the proportion of the whole foreground pixels to entire image pixels as $\omega 0$, the foreground average gray level as $\mu 0$. Put the proportion of the whole background pixels to entire image pixels as $\omega 1$, the background average gray level as $\mu 1$, the total average of the image gray as $\mu$, the variance between classes as g. Set the number of gray levels of pixels in the image which is less than the certain threshold $\mathrm{T}$ as N0, the number of gray levels of pixels in the image which is more than the certain threshold T as N1. The formula is as follows:

$$
\begin{aligned}
& N_{0}+N_{1}=\mathrm{M} \times \mathrm{N} \\
& \omega_{0}=N_{0} /(\mathrm{M} \times \mathrm{N}) \\
& \omega_{1}=N_{1} /(\mathrm{M} \times \mathrm{N}) \\
& \omega_{0}+\omega_{1}=1
\end{aligned}
$$

$$
\omega_{0} \times \mu_{0}+\omega_{1} \times \mu_{1}=\mu
$$

$$
\omega_{0} \times\left(\mu_{0}-\mu\right)^{2}+\omega_{1} \times\left(\mu_{1}-\mu\right)^{2}=g
$$

$$
\omega_{0} \times \omega_{1} \times\left(\mu_{1}-\mu_{0}\right)^{2}=g
$$

We iterate through (7) to get the maximum variance between threshold $\mathrm{T}$ and that is the optimal threshold, and put the threshold $\mathrm{T}$ into the current rule of growth. Now the rule of growth is restricted by the new principle, one is the gray difference is less than the threshold $\mathrm{T}$ and the other is the seed pixel value is less than optimal threshold, which covers the shortage for traditional region growing of the insufficient segmentation or the excessive segmentation.

\section{Calculating area}

In this study, we adopt the analytic method to calculate the area of irregular region [13-14]. It calculates the irregular area by using the coordinates of the inflection point and is suitable for different complex polygon area. The formula is as follows:

$$
\begin{aligned}
& F=\frac{1}{2}\left(\sum_{k=1}^{n} x_{k}\left(\mathrm{y}_{k-1}-\mathrm{y}_{k+1}\right)\right) \\
& F=\frac{1}{2}\left(\sum_{k=1}^{n} y_{k}\left(x_{k-1}-x_{k+1}\right)\right)
\end{aligned}
$$

According to the (8) and (9) above, it shows that the region area is equal to the half of the sum that all points value of abscissa multiplied sequentially by difference the point value that before and after of ordinate, or all points value of ordinate multiplied sequentially by difference the point value that before and after of abscissa. In practice, (8) and (9) can be used separately and check to verify each other.

In this study, we use adaptive seeded region growing algorithm to obtain the lesion region. Here we get a region area, and what we need is the inflection point on the boundary, so the first thing to do is to deal with the region and get the points by processing all the points on the boundary of the lesion region. That's the points we want to process. Generally, the Analytic method can calculate the irregular region by using (8) or (9). Analytic method has a good performance in the measurement of irregular region, but the accuracy of the results will have direct relationship with the position and the number of the inflection point. General analytic method will be in a straight line instead of a curve, when it performs the calculation on the region area, which produces the error on the precision. The less the inflection point found, the worse it will be. That is the denser the boundary point is, the shorter the interval point connection is, the higher the area measurement accuracy is. Therefore, while dealing with the inflection point on the boundary of the lesion area, we are supposed to determine as far as possible that the difference value between two adjacent points is one pixel. In order to improve the accuracy of calculation, we use the (8) and (9) on the same time, and then calculate the average value, so as to reduce the error. 


\section{ANALYSIS OF EXPERIMENTAL RESULTS}

The experimental results are built on Windows 7 by Qt msvc2013 software. The experiment medical images include CT and MR. Here are the experimental results:

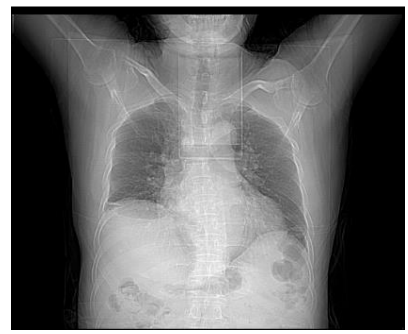

(a)Original image

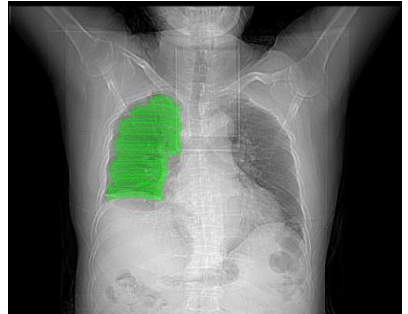

(b)Region growing segmentation image, when the threshold $\mathrm{T}$ is 50

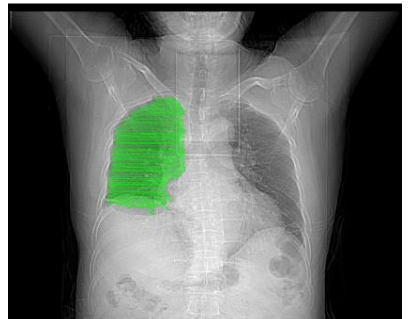

(c) Region growing segmentation image after processing threshold

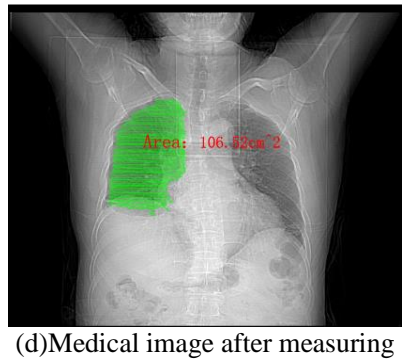

Figure 3. the measurement of CT medical image

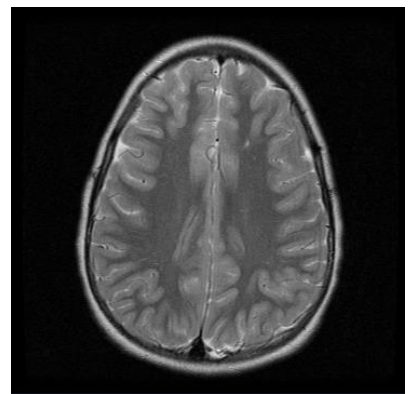

(a)Original image

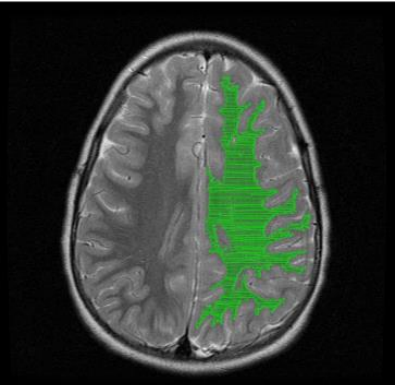

(b) Region growing segmentation image, when the threshold $\mathrm{T}$ is 20

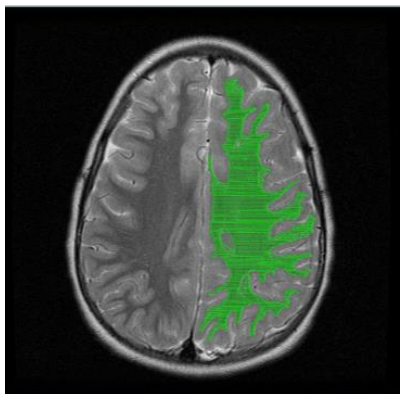

(c) Region growing segmentation image after processing threshold

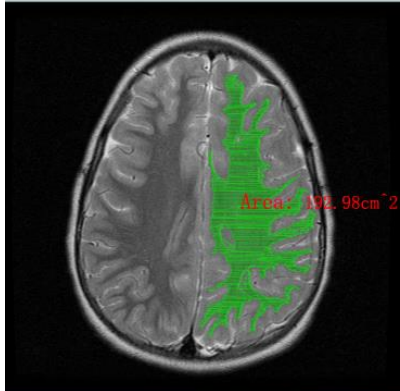

(d) Medical image after measuring

Figure 4. the measurement of MR medical image

In Fig .3(a) is the original CT image, Fig .3(b) is the CT image after region growing segmentation, Fig .3(c) is the CT image after the threshold processing region growing, Fig .3(d) is the CT image after measurement. In Fig .4(a) is the original MR image, Fig .4(b) is the MR image after region growing segmentation, Fig .4(c) is the MR image after the threshold processing region growing, Fig .4(d) is the MR image after measurement. As can be seen from the figure, the original seeded region growing algorithm has the problem of insufficient segmentation. There is significant effect after the threshold processing for traditional region growing method which greatly reduces the probability of the insufficient segmentation or the excessive segmentation. Not only does it improve the accuracy of lesions region, but it also can measure and calculate the lesions area rapidly and accurately. Furthermore, no matter it is CT or MR medical image, this algorithm can figure lesions regions quickly, which greatly saves the preoperative preparation time and fight more for patients.

\section{CONCLUSION}

In this study, we adopt the measurement of the lesions area based on the adaptive seeded region growing algorithm. Compared with the ordinary film and ellipse 
area measurement, this method is able to find out the lesion boundary quickly and calculate the lesion area accurately, which for the doctor, is undoubtedly of great significance. However the disadvantage of seed region growing is that it has impact on the noise and the shade of gray of shadow, which could lead to the insufficient segmentation or the excessive segmentation problem. So how to achieve image filtering [15] as well as combine with other theoretical approaches will be the direction of further research.

\section{REFERENCES}

[1] Christian Ledig et al. Multi-atlas segmentation with augmented features for cardiac MR images. Medical Image Analysis, vol. 19, no.1, Jan. 2015, pp. 98-109

[2] Xiaohong $\mathrm{Hu}$, "Image Segmentation Algorithm of Fracture Tracking Trajectory in Industrial CT Image Management System" Proc. Measuring Technology and Mechatronics Automation (ICMTMA), 2014 Sixth International Conference on, Jan. 2014, pp 319 - 322, doi: 10.1109/ICMTMA.2014.79

[3] Geng-Cheng Lin, Wen-June Wang et al. i Multispectral MR images segmentation based on fuzzy knowledge and modified seeded region growing. Magnetic Resonance Imaging, vol. 30, no.2, Feb. 2012, pp. 230-246

[4] Frank Y. Shih, Shouxian Cheng. Automatic seeded region growing for color image segmentation. Image and Vision Computing, vol. 23, no.10, Sep. 2005, pp. 877-886

[5] Adams R, Bischof L. Seeded region growing. IEEE Transactions on Pattern Analysis and Machine Intelligence, 1997, vol. 18, pp. 1065-1071

[6] Ashish K. Rudra, Ananda S. Chowdhury et al. Kidney segmentation using graph cuts and pixel connectivity. Pattern Recognition Letters, vol. 34, no.13, Oct. 2013, pp. 1470-1475
[7] Hans Luijendijk. Automatic threshold selection using histograms based on the count of 4-connected regions. Pattern Recognition Letters, vol. 12, Apr. 1991, pp. 219-228

[8] Zuoyong Li, Chuancai Liu. Gray level difference-based transition region extraction and thresholding. Computers \& Electrical Engineering, vol. 35, no.5, Sep. 2009, pp. 696-704

[9] J.N. Kapur et al. A new method for gray-level picture thresholding using the entropy of the histogram. Computer Vision, Graphics, and Image Processing, vol. 29, no.4, Mar. 1985, pp. 273-285

[10] uddin Khan Nafis, K.V. Arya et al. Histogram statistics based variance controlled adaptive threshold in anisotropic diffusion for low contrast image enhancement. Signal Processing, vol. 93, no.6, June 2013, pp. 1684-1693

[11] Mengxing Huang, "An Improved Image Segmentation Algorithm Based on the Otsu Method Software Engineering" Proc. Artificial Intelligence, Networking and Parallel \& Distributed Computing (SNPD), 2012 13th ACIS International Conference on, Aug. 2012, pp. 135 - 139, doi: 10.1109/SNPD.2012.26

[12] Liu Jianzhuang, "Automatic thresholding of gray-level pictures using two-dimension Otsu method" Proc. Circuits and Systems, 1991. Conference Proceedings, China, 1991 International Conference on, Jun. 1991, pp.325 - 327 vol.1, doi: 10.1109/CICCAS.1991.184351

[13] Jonathan C. Marshall, Martin L. Hazelton. Boundary kernels for adaptive density estimators on regions with irregular boundaries. Journal of Multivariate Analysis, vol. 101, no.3, Apr. 2010, pp. 949-963

[14] Yu. A. Melnikov, V. Reshniak. A semi-analytical approach to Green's functions for heat equation in regions of irregular shape. Engineering Analysis with Boundary Elements, vol. 46, no.4, Sep. 2014, pp. 108-115

[15] Juan Climent, Luiz S. Oliveira. A new algorithm for number of holes attribute filtering of grey-level images. Pattern Recognition Letters, vol. 53 , no.1, Feb. 2015, pp. 24-30 